\title{
Particle Shape Modification in Comminution ${ }^{\dagger}$
}

\author{
E. Kaya \\ Process Technology Center, \\ Phelps D odge M ining Company* \\ R. Hogg \\ Department of E nergy and Geo-E nvi ronmental \\ Engineering, The Pennsylvania State University** \\ S. R. Kumar \\ Seisint, Inc***
}

\begin{abstract}
The evolution of particle shape during the course of comminution processes has been investigated. Shape is characterized using a variety of quantitative shape descriptors determined from particle profiles obtained by image analysis. Descriptors related to particle elongation, roundness and angularity are emphasized. Distributions of the descriptors have been determined for a range of particle sizes, for differ ent extents of grinding for various equipment types. For a gi ven descriptor, the distributions of measured values generally follow a consistent pattern (often roughly log normal). Typically, the means and standard deviations show progressive changes as grinding time increases. F or the most part, prolonged exposure to the grinding environment leads to rounding of the particles.
\end{abstract}

\section{Introduction}

The importance of particle shape has gained increasing recognition in recent years. It is clear that shape can play a significant role in the use of particles as abrasives and in applications involving packing in powder compacts, slurry rheology, etc. For particles produced by comminution, shape may be determined by material characteristics such as crystal cleavage and by the nature of the breakage process involved. While it is generally recognized that comminution can lead to changes in particle shape, relatively few attempts to quantify these effects have been reported. Furthermore, largely due to the lack of widely accepted measures of shape, there is some disagreement on the evolution of particle shape in grinding processes. For example, B ond [1] considered that the character of the material being broken has more influence on the shape of the product than the type of size reduction machine used. Similarly, Heywood [2] stated that the shape of particles produced on initial

\footnotetext{
* Safford, AZ

** University Park, PA

*** Boca Raton, FL

+ Accepted: September 20, 2002
}

fracture is dependent upon the characteristics of the material. On the other hand, Rose [3] suggested that the type of the mill has the major effect on the particle shape although the properties of the material are also a factor. Similarly, Charles [4] observed that the shape of glass particles produced by a single fracture depended on the rate of application of stress.

The particular mode of breakage is likely to affect the shape of product particles. M assive fracture can be expected to produce highly irregular particles with sharp edges formed by the intersection of propagating cracks. Attrition of particles, by surface erosion or chipping at edges or corners, is more likely to cause rounding of particles although the small fragments removed may be quite irregular in shape. It follows that grinding conditions that favor one breakage mode over another may be critical in determining product particle shape. For any system, the prevailing conditions will generally depend on the type of machine and the properties and size of the particles being broken. Since there are normally distributions of particle sizes and applied stresses, it is reasonable to expect a distribution of product shapes. Gaudin [5] observed that large particles were often subject primarily to attrition, tending to become more rounded in shape, while finer material typically underwent 
massive fracture leading to more angular product partidies. Based on examination of crushed glass partiwcles, Tsubaki and Jimbo [6] concluded that particle shape varies with size.

Holt [7] reviewed the effect of comminution devices on particle shape and concluded that single-pass devices such as roll crushers generally produce angular particles while retention systems such as ball mills produce more rounded particles. Dumm and Hogg [8] showed that the rounding effect in ball mills becomes more pronounced with increased grinding time. Durney and M eloy [9] investigated the shape of particles produced by jaw crushers using Fourier analysis to provide a quantitative description of the shape characteristics. In particular, they compared the results of crushing under single-particle and choke-feeding conditions. They observed that when the particles were fed into the mill one at a time the products were highly angular, while choke feeding produced more "blocky" or rounded particles. In each case, the finer particles had more angular and irregular shapes.

In the present paper, populations of crushed particles from different comminution devices were evaluated using a computerized image analysis technique to quantify particle shape in terms of physically recognizable shape descriptors.

\section{Shape Analysis}

Several different approaches to the analysis of particle shape have been described in the literature. Fourier analysis of the vector of polar coordinates that represent the particle profile yields a set of Fourier coefficients that, in principle, define the shape of the particle [10,11]. M eloy [12] observed simple correlations among the coefficients for a variety of different shapes and defined a two-parameter particle "signature" as an overall characteristic of the particle shape. Durney and M eloy [9] used statistical procedures to detect significant differences in the Fourier coefficients obtained from different populations. Fractal analysis $[13,14]$ has been widely applied to particle shape characterization and is especially attractive for highly complex shapes such as those of agglomerates. The approach adopted in the present work is based on an attempt to define shape in terms of physically recognizable features such as elongation and angularity.

An image processing system was used to provide digitized particle profiles from optical microscopy (or from scanning electron micrographs) by means of a procedure developed by Dumm and Hogg [8] and modified by Kumar [15] and Kaya [16]. A series of straight-line segments was fitted to the set of $\mathrm{N}$ points representing the complete profile as illustrated in Figure 1. The intersections of these linear segments define a reduced set of $n$ perimeter points $(n<<N)$ and describe an $n$-sided polygon that represents the essential features of the original profile.

In order to minimize the errors associated with the use of the fitted polygon, the projected area and centroid of the image were first obtained from the original digitized profile. Designating the $x-y$ coordinates of the original perimeter points as $\left(x_{i}, y_{i}\right)$, the crosssectional area of the particle was determined from:

$$
A=\frac{1}{2} \sum_{i=1}^{N}\left(x_{i+1}-x_{i}\right)\left(y_{i+1}+y_{i}\right)
$$

where the point at $\left(\mathrm{x}_{\mathrm{N}+1}, \mathrm{y}_{\mathrm{N}+1}\right)$ represents the return to the initial starting point $(i=1)$. The coordinates $\bar{x}, \bar{y}$ of the centroid of the image can be obtained from:

$$
\bar{x}=\frac{\sum_{i=1}^{N}\left(x_{i+1}^{2}-x_{i}^{2}\right)\left(y_{i+1}+y_{i}\right)}{2 \sum_{i=1}^{N}\left(x_{i+1}-x_{i}\right)\left(y_{i+1}+y_{i}\right)}
$$

and

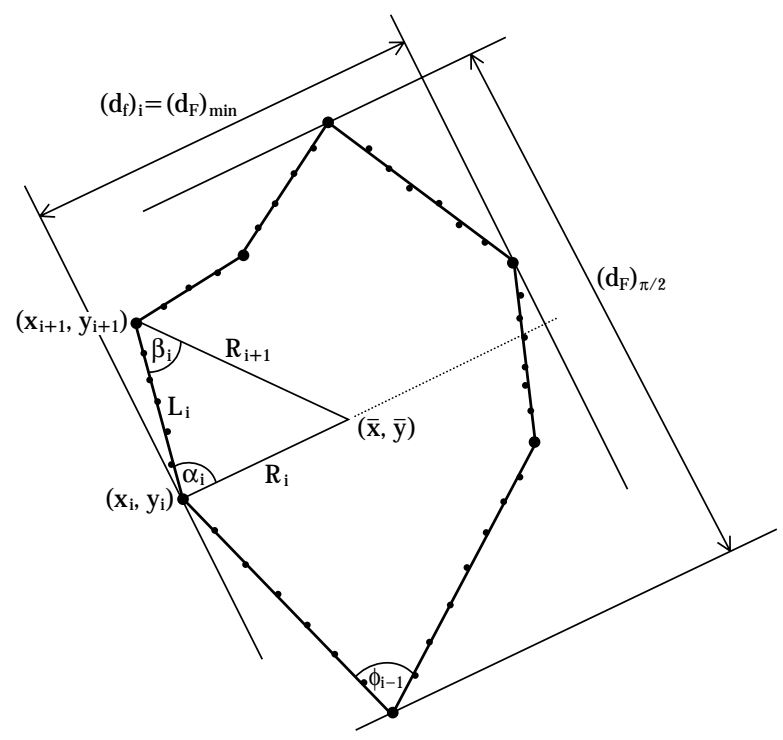

- Original Perimeter Point

- Reduced Perimeter Point

Fig. 1 Representation of a particle profile consisting of $\mathrm{N}$ perimeter points ( $\mathrm{N}=40$ in this example) by a fitted polygon defined by a set of $n$ reduced perimeter points ( $n=7$ in this case). 
The equivalent-circle mean radius of the particle can be calculated using:

$$
\mathrm{R}_{0}=\sqrt{\mathrm{A} / \pi}
$$

The radial vectors $R_{i}$ from the centroid to each of the $\mathrm{n}$ perimeter points on the reduced profile are given by:

$$
R_{i}=\sqrt{\left(x_{i}-\bar{x}\right)^{2}+\left(y_{i}-\bar{y}\right)^{2}}
$$

The angles $\phi_{i}$ between adjacent edges of the fitted polygon were evaluated by applying the cosine rule to the triangles defined by the edge and the adjacent radial vectors as shown in Figure 1. Thus, for the edges intersecting at the perimeter point $\left(x_{i}, y_{i}\right)$ the angle $\alpha_{i}$ is given by:

$$
\cos \alpha_{i}=\frac{R_{i}^{2}+L_{i}^{2}-R_{i+1}^{2}}{2 R_{i} L_{i}}
$$

where $L_{i}$ is the length of the edge connecting points $\left(x_{i}, y_{i}\right)$ and $\left(x_{i+1}, y_{i+1}\right)$ (see Figure 1 ). Similarly, $\beta_{i}$ can be obtained from:

$$
\cos \beta_{i}=\frac{R_{i+1}^{2}+L_{i}^{2}-R_{i}^{2}}{2 R_{i+1} L_{i}}
$$

The angle $\phi_{i}$ is simply the sum: $\alpha_{i}+\beta_{i}$.

Feret's diameters are defined as the distance between two parallel lines tangent to opposite sides of a particle, in some particular orientation. For any perimeter point, the corresponding Feret's diameter, $\left(d_{f}\right)_{i}$ can be obtained by projecting the other perimeter points on to the vector $R_{i}$ and determining the maximum distance from the original point (see Figure 1 ).

The following shape descriptors were defined to represent specific geometric features of the profile.

1) The elongation $E$ was defined using the ratio of the minimum Feret's diameter to that at right angles to it. Thus,

$$
E=\left[\left(d_{F}\right)_{\pi / 2} /\left(d_{F}\right)_{\min }\right]-1
$$

where $\left(d_{F}\right)_{\min }$ is the minimum of the set of measured Feret's diameters and $\left(d_{F}\right)_{\pi / 2}$ is the Feret's diameter measured perpendicular to $\left(d_{F}\right)_{\min }$. As defined, the elongation is zero for a circular profile.

2) The angular variability $V_{\phi}$ was defined to represent the variation in the angles $\phi_{i}$ between adjacent edges on the reduced profile. Specifically, for $\phi_{i}$ expressed in radians,

$$
\mathrm{V}_{\phi}=\sum_{\mathrm{i}=1}^{\mathrm{n}}\left(1-\frac{\phi_{\mathrm{i}}}{\pi}\right)^{3}
$$

The third power was used in order to emphasize the role of the smaller angles, which are considered to contribute the most to the "angularity" of a particle [15]. A many-sided polygon fitted to a circle gives a set of angles close to $\pi$ with a corresponding angular variability close to zero.

3) The radial variability $V_{R}$ was used to describe the departure of the profile from a circle. The particular definition used was

$$
\mathrm{V}_{\mathrm{R}}=\sum_{\mathrm{i}=1}^{\mathrm{n}}\left|\frac{\mathrm{R}_{\mathrm{i}}}{\mathrm{R}_{0}}-1\right|
$$

where $R_{0}$ is the equivalent spherical diameter as defined by Equation 4 and the $R_{i}$ are the lengths of the radial vectors from the centroid to each of the $n$ perimeter points (Equation 5). Since each $R_{i}$ would be equal to $R_{0}$ for a circle, the radial variability would be

\begin{tabular}{|c|c|c|c|}
\hline \multirow[t]{2}{*}{$\begin{array}{l}\text { Reduced Perimeter } \\
\text { Point, i } \\
\text { (clockwise from top) }\end{array}$} & \multirow[t]{2}{*}{$\begin{array}{c}\text { Relative Distance } \\
\text { from Centroid, } \\
\mathbf{R}_{\mathbf{i}} / \mathbf{R}_{\mathbf{0}}\end{array}$} & \multicolumn{2}{|c|}{$\begin{array}{c}\text { Angle between } \\
\text { Adjacent Faces, } \\
\phi_{i}\end{array}$} \\
\hline & & Radians & Degrees \\
\hline 1 & 1.409 & 1.479 & 84.7 \\
\hline 2 & 1.070 & 2.336 & 133.8 \\
\hline 3 & 0.885 & 2.549 & 146.0 \\
\hline 4 & 1.454 & 1.227 & 70.3 \\
\hline 5 & 0.878 & 2.656 & 152.2 \\
\hline 6 & 1.111 & 1.855 & 106.3 \\
\hline 7 & 0.915 & 3.607 & 206.7 \\
\hline
\end{tabular}
zero.

Calculated values of the various parameters are given in Table 1 for the schematic profile shown in Figure 1.

Table 1 Relative dimensions and calculated shape descriptors for the schematic particle profile shown in Figure $\mathbf{1}$.

Shape Parameters:

Elongation, $\mathrm{E}: \quad 0.41$

Radial Variability, $\mathrm{V}_{\mathrm{R}}: \quad 1.37$

Angular Variability, $\mathrm{V}_{\phi}: 0.47$

\section{Experimental Systems}

Shape descriptors were measured for a range of particle sizes produced by crushing and grinding under a variety of conditions. Approximately 600 particles were analyzed from each population and the distribution of each descriptor was evaluated. The 
קי

materiars used were a high-volatile bituminous coal from a continuous mining operation in the Pittsburgh WCoal Seam (Green Country, PA) and quartz from $\mathrm{N}$ or th Carolina. The following crushing and grinding devices were used to produce particles in different size ranges:

- Jaw Crusher - used to reduce feed materials about $1 \mathrm{~cm}$ in size to less than about $5 \mathrm{~mm}$

- Hammer Mill (Holmes pulverizer) - used to crush coal particles in the $5 \mathrm{~mm}$ to $1 \mathrm{~cm}$ size range to less than $1 \mathrm{~mm}$

- Disk Mill (Quaker City) - used to pulverize 1 mm feed particles

- Ring and Puck Mill (Bleuler Pulverizer) - used for fine grinding of disk mill product to micron sizes

- Planetary Ball M ill (Retsch) - also used for fine grinding of disk mill product.

The jaw crusher, hammer mill and disk mill are essentially single-pass devices, although some, lim-

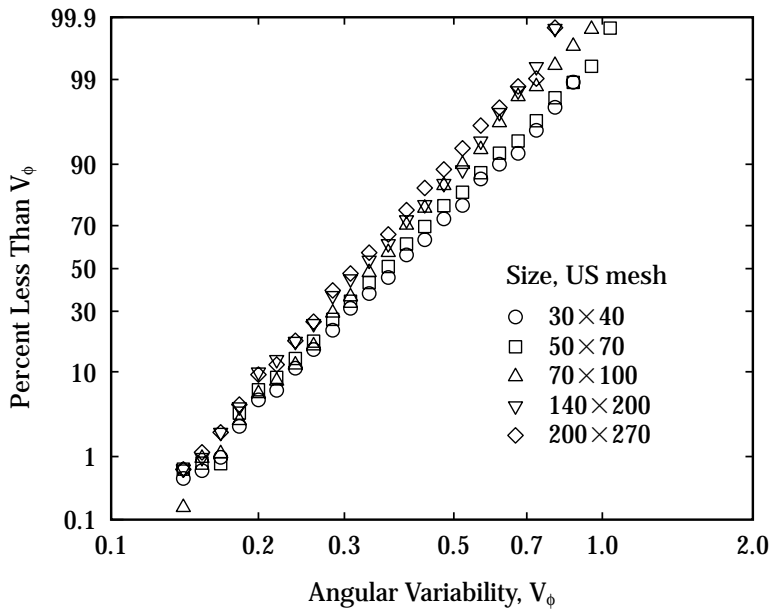

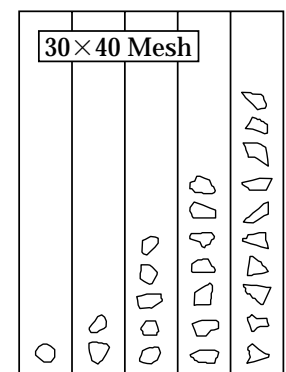

$\begin{array}{llllll}0.10 & 0.19 & 0.25 & 0.32 & 0.42 & 1.00\end{array}$

Angular Variability

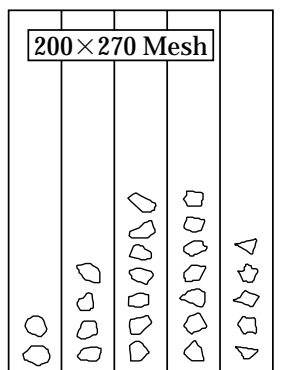

$\begin{array}{llllll}0.10 & 0.19 & 0.25 & 0.32 & 0.42 & 1.00\end{array}$

Angular Variability
Fig. 2 Graphical and visual representations of the number distributions of angular variability for coal particles produced in a jaw crusher (feed size $9.5 \times 12.7 \mathrm{~mm}$; gap setting $6.4 \mathrm{~mm}$ ). ited retention of broken material may occur. The ringand-puck and planetary mills are retention devices that can subject particles to repeated breakage. In the case of the latter two devices, different grinding times were used to vary the exposure of particles to the grinding environment.

\section{Experimental Results}

\section{Analysis of Shape for Different Materials}

In order to evaluate particle shape effects for different materials, samples of the coal and quartz were prepared as $9.5 \times 12.7 \mathrm{~mm}$ size fractions and fed to a jaw crusher with a gap setting of $6.4 \mathrm{~mm}$. Products in different size classes $(30 \times 40,50 \times 70,70 \times 100,140 \times$ 200 and $200 \times 270$ US mesh) were analyzed using digitized images obtained by optical microscopy.

Examples of the distributions of angular variability for coal and quartz particles of different sizes are presented in Figures $\mathbf{2}$ and 3. In each case, the distribu-

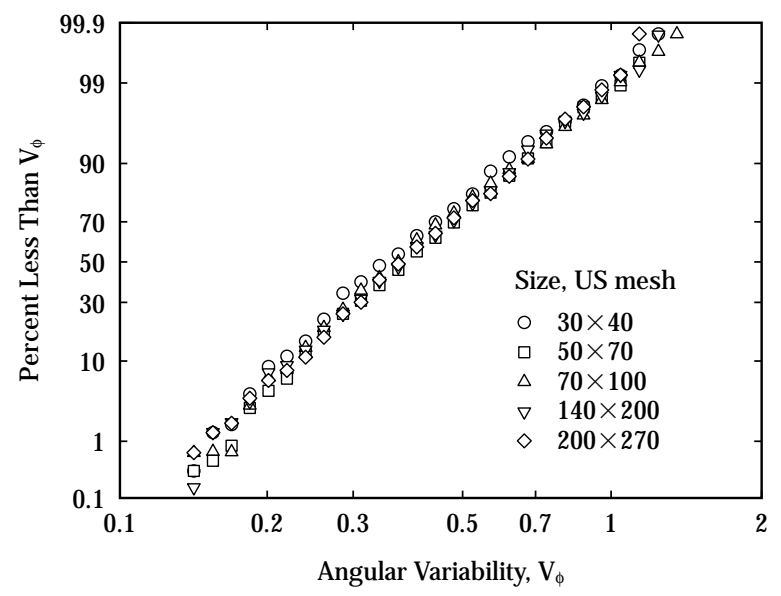

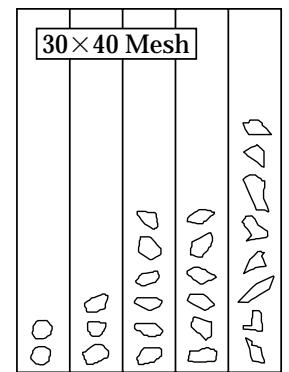

$\begin{array}{llllll}0.10 & 0.19 & 0.25 & 0.32 & 0.42 & 1.00\end{array}$ Angular Variability

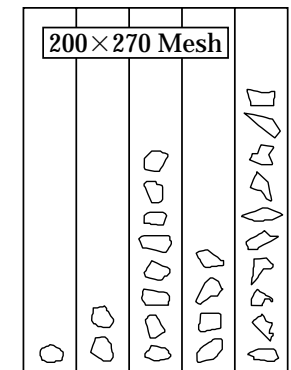

$\begin{array}{llllll}0.10 & 0.19 & 0.25 & 0.32 & 0.42 & 1.00\end{array}$

Angular Variability
Fig. 3 Graphical and visual representations of the number distributions of angular variability for quartz particles produced in a jaw crusher (feed size $9.5 \times 12.7 \mathrm{~mm}$; gap setting 6.4 $\mathrm{mm})$. 
tionsare shown graphically as cumulative plots and a visual kepresentation to illustrate the significance of the variations is included. The particles in each column are typical of that range of angular variability while the number of particles in each column represents the number fraction in that range. The results indicate that, for coal particles, the distributions generally become somewhat narrower and shift to lower values as size decreases, implying a size dependency of the shape. On the other hand, the distributions of angular variability for the quartz particles show no clear systematic variation with size. Very similar trends were observed for the other shape descriptors: elongation and radial variability. It is interesting to note that the distributions appear to conform quite closely to the log-normal distribution.

\section{E ffect of Grinding Procedure}

The particle shape is expected to be affected by the type of machine, by the specific breakage mechanism in a grinding device and by the time spent in the grinding environment. The effect of grinding device on shape was analyzed for coal particles using the Bleuler ring-and-puck pulverizer and the planetary ball mill. The grinding times were set so as to give a similar extent of grinding for each mill. The quantity of feed ( -10 mesh) was $20 \mathrm{~g}$ for the Bleuler and $9.5 \mathrm{~g}$ for the planetary mill. The distributions of elongation are presented in Figure 4. The graph indicates that different grinding devices affect shape differently. Shape does not change substantially with grinding time in a high-energy mill (Bleuler). On the other hand, the shape distributions of the particles produced in the planetary mill shifted towards lower values, indicating that particles become more rounded with increased grinding time. Similar trends have been observed for particle sizes in the size range from 3 to $5 \mu \mathrm{m}$.

Examples of the distributions of angular variability for $70 \times 100$ US mesh coal obtained from a single pass through different crushing and grinding devices are shown in Figure 5. It appears that the jaw crusher, for which massive fracture is the dominant breakage mechanism, produces the most irregular particles. The Quaker City mill, for which most of the breakage is probably by massive fracture of coarse feed particles, also produces quite irregular particles. The Holmes pulverizer, which allows some retention of material and may include contributions from the attrition-type mechanisms, and the Bleuler mill (30 second grinding time), generally produce particles of more regular shape. Similar trends have been ob-

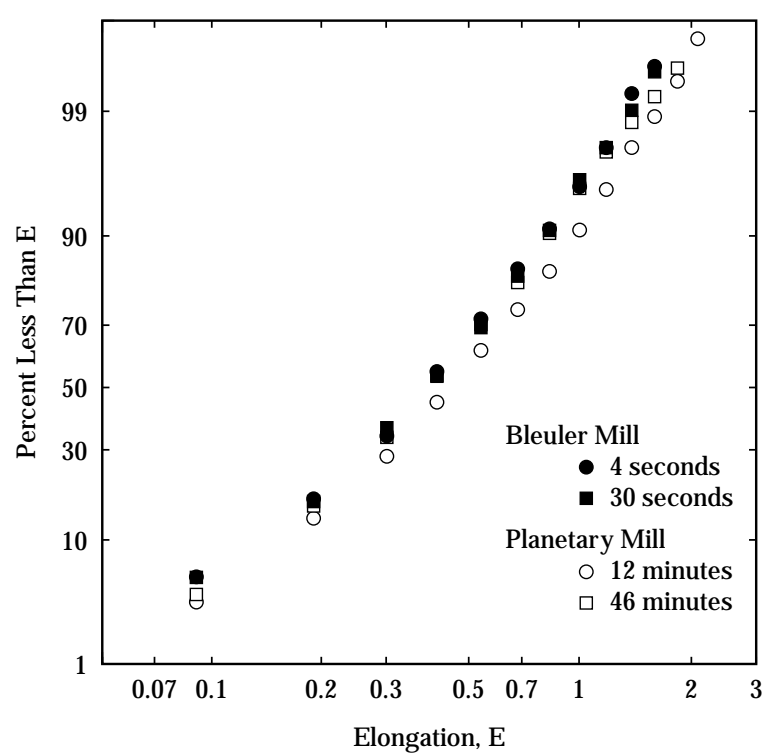

Fig. 4 Number distributions of elongation for coal particles $(200 \times 270$ US mesh) produced in both the Bleuler and planetary mills using different grinding times.

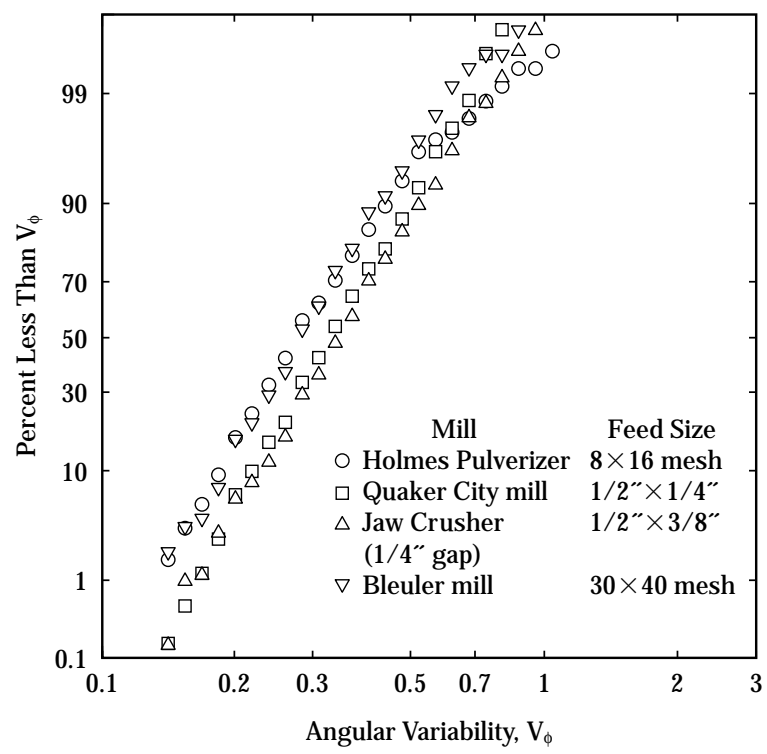

Fig. 5 Number distributions of angular variability for coal particles $(70 \times 100$ US mesh) produced by different comminution devices.

served for $200 \times 270$ US mesh fractions. Changes in feed size to the devices did not lead to significant differences in product particle shape.

The effects of mill type on the average particle shape for different coal product sizes are illustrated in Figure 6. While the variations are generally small, 

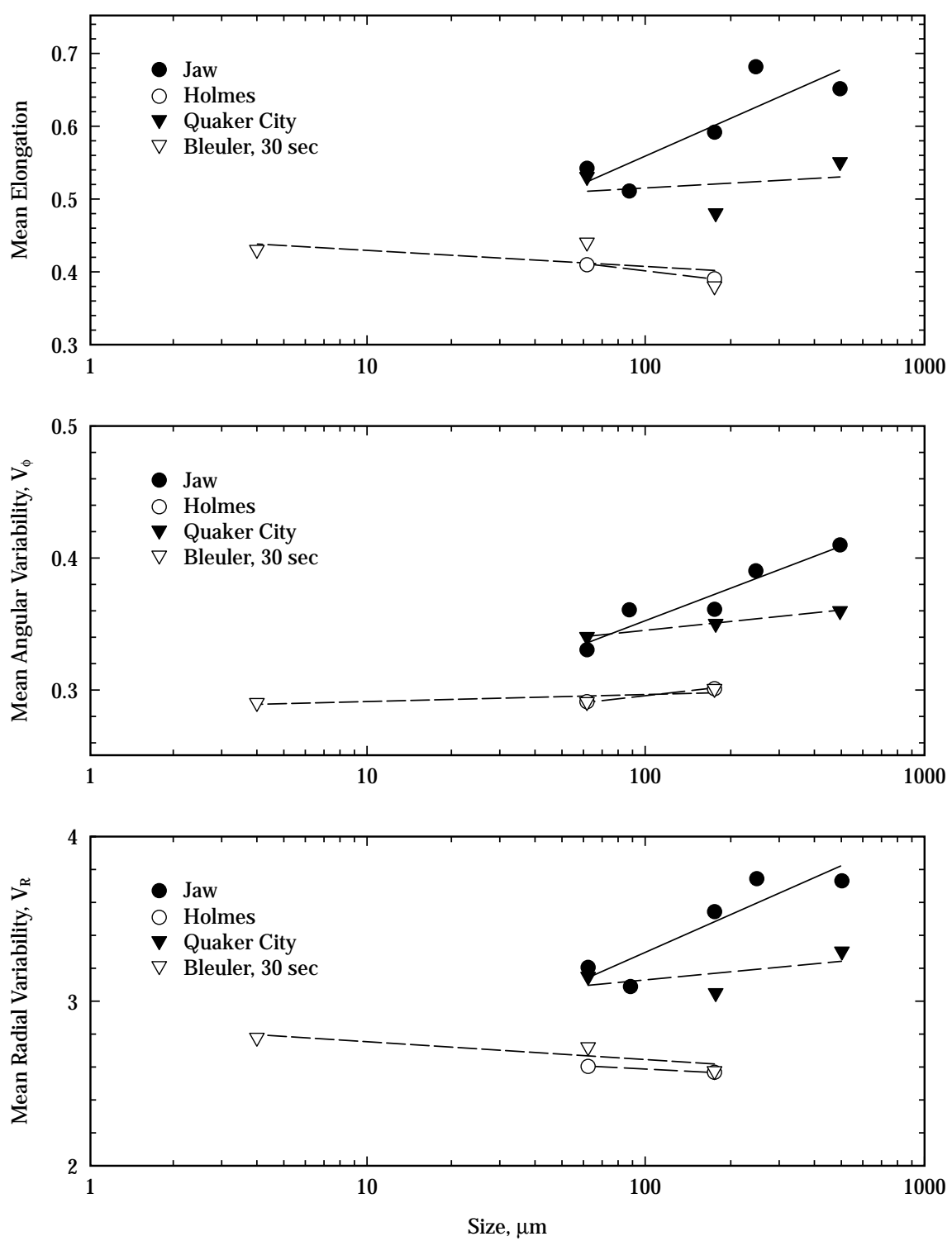

Fig. 6 Variation in shape with particle size for coal ground in different mill types.

the trends are consistent and similar for each of the three descriptors. Large particles produced by single breakage events tend to be quite irregular in shape while particles subject to repeated breakage or long exposure to the grinding environment are usually more rounded.

\section{Conclusions}

The results of this investigation indicate that, for the materials studied (quartz and coal), the shape of particles produced by size reduction is controlled by

a) the nature of the material being reduced

b) the type of comminution device used and the predominant breakage mechanisms involved

c) the time spent in the grinding environment. In particular, it is concluded that the products of individual breakage events are typically angular and irregular in shape. Continued exposure to the grinding environment leads to rounding of the particles. For a given product size distribution, devices that employ high energy input yield products containing a high proportion of newly-created particles. Such devices, therefore, favor the production of irregular particles. Grinding machines for which the energy input is relatively low, on the other hand, rely on repeated breakage action for size reduction and tend to produce more rounded product particles. 
Thordifferences in the size dependence of productarattide shape for coal and quartz suggest that the existence of structural features such as cleats in coal or cleavage planes in crystals may lead to more angular and irregular products of comminution. Furthermore, since the effect appears to be more pronounced for low-energy devices, it may be possible to achieve some degree of control over shape through appropriate equipment selection. However, considerable additional work would be needed to establish the basis for such control.

\section{Acknowledgements}

The work described in this paper was supported in part under the M ineral Institutes Program by Grant Nos. G1105142, G1115142 and G1125142 from the Bureau of M ines, US Department of the Interior, as part of the Generic Mineral Technology Center for Respirable Dust.

\section{Nomenclature}

A : Cross-sectional area

$\left(d_{f}\right)_{i} \quad:$ Feret's diameter corresponding to perimeter point $\mathrm{i}$

$[\mathrm{m}]$

$\left(d_{f}\right)_{\min }$ : M inimum Feret's diameter of a particle $[\mathrm{m}]$

$\left(d_{f}\right)_{\pi / 2}:$ Feret's diameter perpendicular to $\left(d_{f}\right)_{\min }[\mathrm{m}]$

E : Elongation of a particle [-]

$\mathrm{L}_{i} \quad$ : Edge-length on a particle profile

[m]

$\mathrm{n} \quad$ : Number of reduced perimeter points on a fitted particle profile

$\mathrm{N} \quad$ : N umber of measured perimeter points on a particle profile

$\mathrm{R}_{0} \quad$ : Equivalent-circle mean particle radius

$\mathrm{R}_{\mathrm{i}} \quad$ : Length of radial vector from particle centroid to the $i^{\text {th }}$ perimeter point

$V_{R} \quad$ : Radial variability

$V_{\phi} \quad$ : Angular variability

$x_{i}, y_{i}:$ Cartesian coordinates of the $i^{\text {th }}$ perimeter point

$\bar{x}, \bar{y} \quad$ : Cartesian coordinates of a particle centroid

$\alpha_{i} \quad$ : Angle defined in Figure 1

[radians]

$\beta_{\mathrm{i}} \quad$ : Angle defined in Figure 1

[radians]

$\phi_{\mathrm{i}} \quad$ : Angle between adjacent edges on a particle profile

[radians]

\section{References}

1) Bond, F. C., "Control Particle Shape and Size," Chemical Engineering, pp. 195-198 (Aug. 1954).

2) Heywood, H., "Powder Production by Fine Milling," in Powders in Industry, Society of Chemical Industry, pp. 25-26 (1962).

3) Rose, H. E., "Particle Shape, Size and Surface Area," in Powders in Industry, Soc. Chem. Ind., pp. 130-149 (1961).

4) Charles, R. J., "High Velocity Impact in Comminution," M ining Engineering, pp. 1028-1032 (1956).

5) Gaudin, A. M., "An Investigation of Crushing Phenomena," Trans AIM E, 73. pp. 253-317 (1926).

6) Tsubaki, J. and Jimbo, G., "Identification of Particles Using Diagrams and Distributions of Shape Indices," Powder Technology, 22. pp. 171-178 (1979).

7) Holt, C. B., "The Shape of Particles Produced by Comminution - A Review," Powder Technology, 28. pp. 5963 (1981).

8) Dumm, T. F. and Hogg, R., "Characterization of Par ticle Shape," Proceedings of International Symposium on Respirable Dust in the Mineral Industries, SME, Littleton, CO, pp. 283-288, (1990).

9) Durney, T. E. and M eloy, T. P., "Particle Shape Effects due to Crushing Method and Size," Intl. J. Miner. Process, 16. pp. 109-123 (1986).

10) Beddow, J. K. and Meloy, T. P., Advanced Particulate M orphology, CRC Press, B oca Raton FL (1977).

11) Meloy, T. P., "Particulate Characterization: Future Approaches," Chapter 3 in Handbook of Powder Science and Technology, M. E. Fayed and L. Otten, editors, van N ostrand Rheinhold, New York, pp. 69-98 (1984).

12) M eloy, T. P., "Fast Fourier Transforms Applied to Shape Analysis of Particle Silhouettes to Obtain M orphological Data," Powder Technology, 17. pp. 27-35 (1977).

13) $M$ andelbrot, B. P., Fractals: Form, Chance and Dimension, W. H. Freeman, San Francisco (1977).

14) Kaye, B. H., "The description of T wo-Dimensional Rugged boundaries in Fineparticle Science by Means of Fractal Dimensions'," Powder Technology, 46. pp. 245254 (1986).

15) Kumar, S. R., "Characterization of Particle Shape," M . S. Thesis, The Pennsylvania State University (1996).

16) Kaya, E., "Control of Particle Characteristics in the Production of Fine Powder By Grinding," Ph.D. Thesis, The Pennsylvania State University (1996). 


\section{Author's short biography}

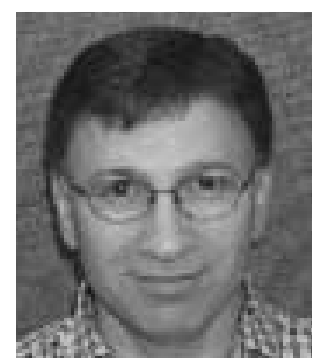

\section{Erol Kaya}

Erol Kaya is currently a Senior Research Engineer at the Process Technology Center, Phelps Dodge M ining Company. He received a B. Sc. degree in mining engineering from the Istanbul Technical University, the M.S. and Ph.D. degrees in mineral processing and $M$. Eng. in environmental pollution control from the Pennsylvania State University. His research interests include particulate technology, grinding and copper flotation.

\section{Richard Hogg}

Richard Hogg is Professor of Mineral Processing and GeoEnvironmental Engineering at the Pennsylvania State University. He received a B.Sc. from the University of Leeds and the M.S. and Ph.D. degrees from the University of California at Berkeley. Dr Hogg's research interests include fine particle processing, particle characterization, and colloid and surface chemistry.

\section{Senthil Kumar}

Senthil Kumar graduated from the Pennsylvania State University with an M.S. in Mineral Processing and an MEng. in Computer Science and Engineering. He received his $B T e c h$. in $M$ etallurgical engineering from the Indian Institute of Technology, Bombay. He is presently a technical architect at Seisint Inc., a software company in Boca Raton, Florida. 